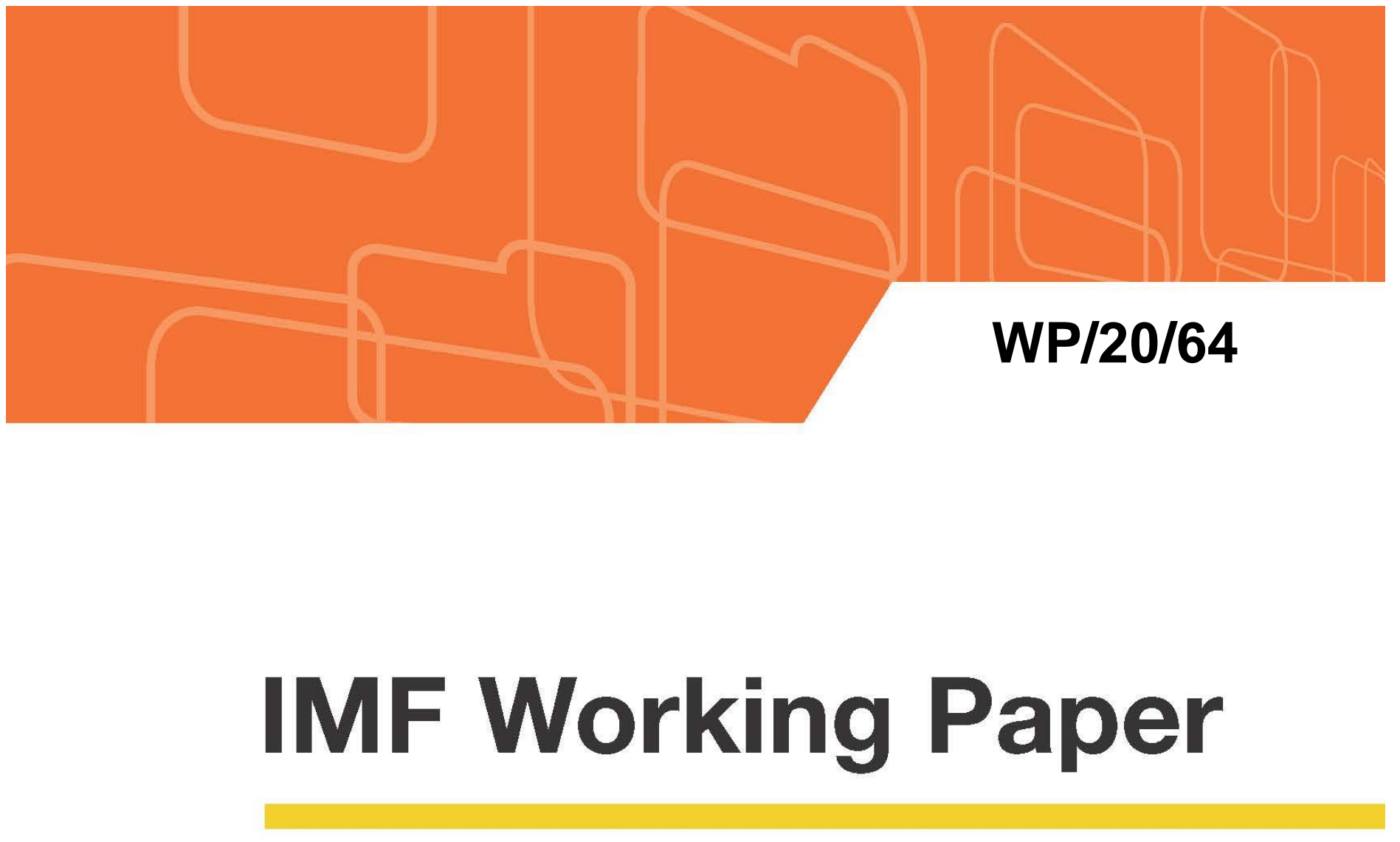

\title{
Model-Based Globally-Consistent Risk Assessment
}

by Michal Andrle and Benjamin Hunt

IMF Working Papers describe research in progress by the author(s) and are published to elicit comments and to encourage debate. The views expressed in IMF Working Papers are those of the author(s) and do not necessarily represent the views of the IMF, its Executive Board, or IMF management. 


\title{
IMF Working Paper
}

Research Department

\section{Model-Based Globally-Consistent Risk Assessment ${ }^{1}$ \\ Prepared by Michal Andrle and Benjamin Hunt}

Authorized for distribution by Benjamin Hunt

May 2020

\section{IMF Working Papers describe research in progress by the author(s) and are published to elicit comments and to encourage debate. The views expressed in IMF Working Papers are those of the author(s) and do not necessarily represent the views of the IMF, its Executive Board, or IMF management.}

\begin{abstract}
This paper outlines an approach to assess uncertainty around a forecast baseline as well as the impact of alternative policy rules on macro variability. The approach allows for nonGaussian shock distributions and non-linear underlying macroeconomic models. Consequently, the resulting distributions for macroeconomic variables can exhibit skewness and fat tails. Several applications are presented that illustrate the practical implementation of the technique including confidence bands around a baseline forecast, the probabilities of global growth falling below a specified threshold, and the impact of alternative fiscal policy reactions functions on macro variability.

JEL Classification Numbers: C6, E3, E52, E62

Keywords: DSGE models, predictive density, nonlinear, non-Gaussian, skew, fat tails Author's E-Mail Address: mandrle@imf.org, bhunt@imf.org
\end{abstract}

\footnotetext{
${ }^{1}$ We would like to thank Jan Brůha, Mika Kortelainen, Miroslav Plašil, Rafael Portillo, Antti Ripatti, Malik Sheheryar, Kadir Tanyeri, Fan Zhang, and (with special thanks) Anders Warne for useful discussions. All errors and omissions are of the authors.
} 


\section{Contents}

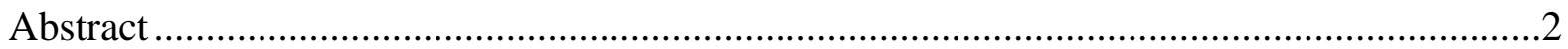

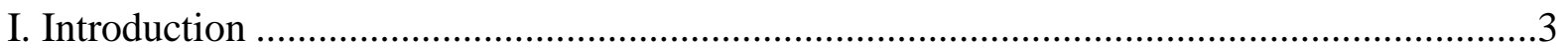

II. Constructing Model-Based Predictive Distributions.................................................

A. Solving and Simulating Large-Scale Non-Linear Models ..................................5

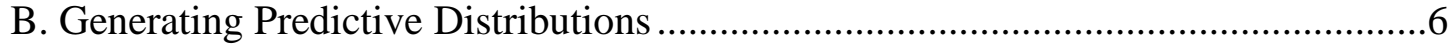

C. Estimating the Structural Economic Shocks with Non-Linear Models .................... 7

"One-to-one": an important special case .................................................... 7

D. Distribution of Shocks and Resampling........................................................

Kernel Density Estimation and Sampling ...............................................

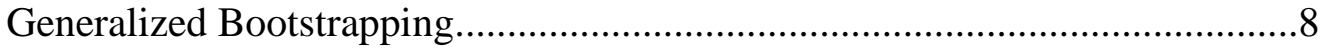

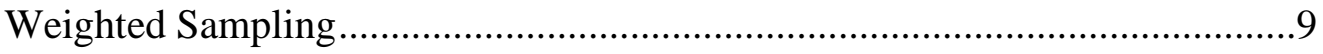

III. Providing Globally-Consistent Risk Assessment ..................................................

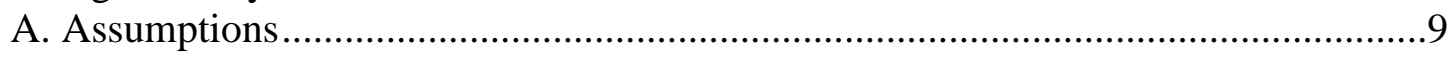

B. Predictive Distributions around the Baseline Forecast ....................................11

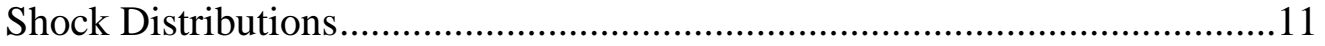

Non-linearity ................................................................................... 11

Output Distributions and "Fan Charts" ......................................................11

C. Effects of Alternative Economic Policies on Predictive Distributions ...................17

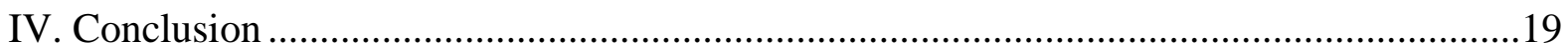

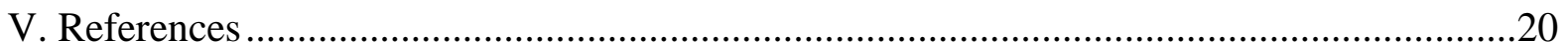

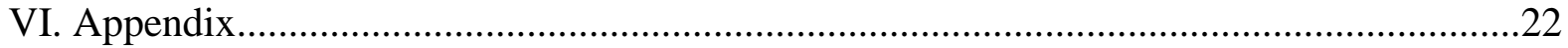

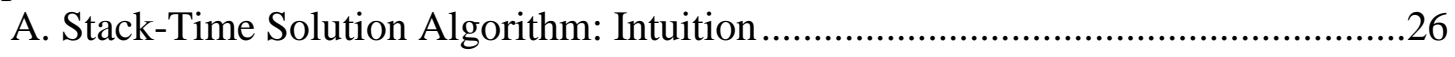

Figures

1: Sample Shocks vs. the Normal Distribution..............................................................13

2: Estimated GDP Growth Distribution (1Y and 5Y horizon) ........................................14

3: Fan Charts around the Baseline Forecast (5-95 percentiles): Japan ...............................15

4: Marginal Distribution of the GDP Growth in the U.S. ................................................16

5: Effects of Economic Policy on Risk Assessment ........................................................18

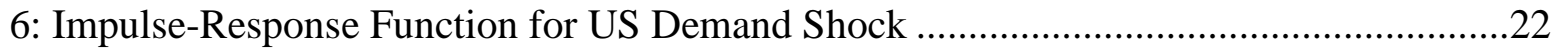

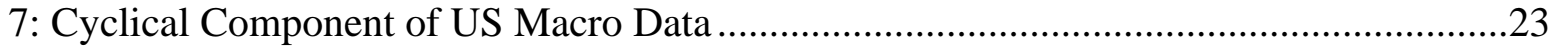

8: Model-implied vs. Actual Outcome.................................................................................24

9: Distribution of GDP Growth and Debt-to-GDP using the Empirical Shock Distribution...25

Table

1: Probability of World GDP Growth of less than 2.5 Percent $\ldots \ldots \ldots \ldots \ldots \ldots \ldots \ldots \ldots \ldots \ldots$

References....................................................................20 


\section{INTRODUCTION}

This paper introduces a practical way of providing model-based risk and policy assessment using stochastic simulations of large-scale, global, and non-linear dynamic economic models. The result of the analysis is a well-defined and globally-consistent predictive distribution of key macroeconomic variables in all countries and regions considered. The distribution of risks depends on the state of the economy-position in the business cycle, or monetary policy space - and its interaction with the model's nonlinearities (zero lower bound, nonlinear Phillips curve, or asymmetric decision rules). The distribution of risks also varies with changes to economic policies, providing a vital insight for assessing alternative policy recommendations.

The benefits of risk assessment using a structural model are immense. The model dynamics have a clear, economically-meaningful interpretation, depend on the initial and the expected state of the economy. The joint distribution of all the model's variables is consistent both within each country and internationally across countries and regions, accounting for existing financial and trade spillovers. A large set of variables can be analyzed, ranging from the real side of the economy, to fiscal and monetary variables. The model-based distributions can help to gauge the probability of recession, the probability of deflation, or the probability of hitting a government debt ceiling, for instance.

In this paper, the focus is on constructing realistic risk and policy assessment by (i) using structural non-linear models and by (ii) using a non-Gaussian distribution of economic shocks, estimated using the past data for the global economy. Both the non-linearity and nonNormality of the shocks allow the models to describe the moments of the data. The resulting predictive distributions vary with the state of the economy and are usually skewed and have fat tails.

This technique for risk assessment is illustrated using the IMF's G20MOD, a module of the Flexible System of Global Models (FSGM, see Andrle et al. 2015). For the policy work, the proposed approach has also been used with the IMF's Global Integrated Monetary and Fiscal model (GIMF, Kumhof et al. 2010) and the IMF's quarterly Global Projection Model (GPM, Carabenciov et al. 2013). All three models are non-linear and, importantly, respect the effective lower bound (ELB) for the nominal interest rates.

The first part of the paper is theoretical. To implement the stochastic simulations, we propose an agnostic and flexible way to estimate the structural economic shocks using non-linear models and to sample from the estimated distribution of the shocks. Crucially, the proposed method accounts for possible dependence among the shocks, which — if ignored — may bias the risk assessment.

The second part of the paper provides two examples. The first example uses the IMF's G20MOD to illustrate the output growth distribution estimates for G20 countries for one-year 
and five-year horizons, forecast "fan charts", suitable to assess the risks of recession or deflation, and the probability of World output growth falling below 2.5 percent. The second example illustrates how the distribution of output growth changes for an economy close to its effective lower bound on interest rates with or without an asymmetric cyclical fiscal policy reaction function.

\section{Constructing Model-Based Predictive Distributions}

To construct the model-based forecast, the model needs to be simulated over the forecast horizon applying appropriate judgment, and the economic shocks and their probability distributions must be determined from the historical data and the structure of the model. The predictive distribution is then constructed using those shock probability distributions to perform stochastic simulations of the model.

Conditional on the deterministic part of the model, the key to realistic predictive distributions are the stochastic assumptions about the economic shocks. It is common to assume that economic shocks are independent and Normally distributed. The independence of structural shocks guarantees the interpretability of the impulse-response functions, etc.

For a risk assessment with a structural model, the assumption of independent Normal distributions can be restrictive, given the observed departures of macroeconomic data from Normality - recessions are deeper then booms, deviations of unemployment above the trend are larger than below the trend, crises are sudden and, thankfully, infrequent - see Morley and Piger (2012), for instance.

The specification of the shock distributions and the model dynamics are intrinsically linked. Those aspects of the observed data that cannot be explained by the model dynamics, will be provided by the shock distributions to replicate the distribution of the observed data.

Deviations from the independent Normal distribution - if they occur - can and should be analyzed and can provide directions for improving the internal model propagation of shocks. However, insisting on independent Normal distribution of economic shocks for construction of the predictive distribution may severely distort the resulting distributions. While the assumptions of independent-Normal shock distributions are prevalent in the literature, the estimated shock realizations are often anything but.

Our solution is to use the non-linear economic model and its dynamics to back out the shocks that, when simulated using the model, will replicate the available observations. Using these estimated shocks, we obtain a non-parametric estimate of their unknown multi-variate distributions. Draws sampled from this distribution and propagated through the model from the initial conditions of the economy are the building blocks for constructing the predictive distributions. 
The rest of the section focuses on the important implementation details - model solution, estimation of economic shocks with a non-linear model, estimation of the shock distributions, and how to sample from such distributions.

\section{A. Solving and Simulating Large-Scale Non-Linear Models}

We consider dynamic forward-looking models of the form

$$
E\left[M\left(x_{t+1 \mid t}, x_{t}, x_{t-1}, \varepsilon_{t} ; \theta\right)\right]=0
$$

where the model variables, $x_{t}$, are driven by their previous state, $x_{t-1}$, structural shocks innovations, $\varepsilon_{t}$, and expectations of future states of the economy, $x_{t+1 \mid t}$. The structural parameters describing the model economy are collected in the parameter vector $\theta$. This is a general specification and most DSGE models in the literature conform to this form.

The large-scale macroeconomic models considered in this paper can be cast, implicitly, into the following reduced form, where expectations have been solved-out:

$$
\begin{gathered}
x_{t}=f\left(x_{t-1}, \varepsilon_{t} ; \theta\right)=g\left(\varepsilon_{t}, \varepsilon_{t-1}, \ldots, \varepsilon_{0}, x_{0} ; \theta\right) \\
y_{t}=\mathrm{S} x_{t}
\end{gathered}
$$

The model's dynamics in period $t$ are then fully described by the economy's initial state, $x_{t-1}$, and the realized structural shocks. Only a subset of the model's variables is observed or observable and these variables are selected by the selection matrix $S$ into the observed variables vector $y_{t}$.

Most large-scale DSGE models are solved under the assumption of "certainty equivalence". This is because (i) the models are either (log)-linearized, or (ii) the models are kept nonlinear but solved as deterministic models, where higher order moments of shock distributions (variance, skewness, etc.) do not enter agents' decisions. ${ }^{2}$

The IMF's global economic models are solved in their non-linear form under certainty equivalence assumption, using a variant of the "stack-time" algorithm, see Juillard (1997). ${ }^{3}$ The consequence of this solution approach is that the distributions of the structural shocks need not be defined beyond their mean, which is zero by assumption.

\footnotetext{
${ }^{2}$ It also means that Jensen's inequality does not hold in these models and thus $\mathrm{E}(\mathrm{f}(\mathrm{x}))=\mathrm{f}(\mathrm{E}(\mathrm{x}))$.

${ }^{3}$ A Dynare toolbox supports the solution method; in fact, it was the first solution method in Dynare. Portable Troll features robust versions of the stacked time algorithm.
} 
While there are drawbacks of the certainty-equivalence solution methods, the very assumption that the higher moments of the shock distribution do not affect the model solution opens the door to the agnostic estimation of the shock distributions below.

See the appendix for more intuition of the "stack-time" non-linear solution algorithm.

\section{B. Generating Predictive Distributions}

Given the model, $f(. ; \theta)$, the goal is to create a projection of future economic dynamics and to assess the risks around the projection. This is a well-known and standard problem in the literature, focused on generating the predictive distribution by sampling the forecast paths using the distribution of the economic shocks $\varepsilon_{t} \sim F_{\varepsilon}($.$) , conditional on the model's structure$ and parameters and the initial conditions of the economy. ${ }^{4}$

For each draw, $\varepsilon_{t, s}$, for $s=1, \ldots, \mathcal{S}$ and $\mathrm{t}=1, \ldots, T$ from the shock distribution, $F_{\varepsilon}($.$) ,$ simulate the model

$$
x_{t, s}=f\left(x_{t-1, s}, \varepsilon_{t, s} ; \theta\right)
$$

In most of the literature, DSGE models are solved using first-order perturbation methods (log-linearized) and with the assumption of structural shock innovations being Gaussian. In such a setup, the predictive distribution of the model's variables is Gaussian as well and analytically tractable, unless parameter uncertainty is considered. ${ }^{5}$

When the shock distribution, $F_{\varepsilon}($.$) , is not Gaussian and/or the model f($.$) is non-linear, the$ joint predictive distribution of $x_{t}$ is generally unknown and can take quite a nontrivial form with asymmetries and fat tails.

Depending on the model, the computational burden of generating the model's predictive distribution can be sizeable, especially when the model is non-linear or when parameter uncertainty is considered. Fortunately, the sampling is "an embarrassingly parallel" problem and the gains from parallel computations are large. ${ }^{6}$

\footnotetext{
${ }^{4}$ We ignore parameter uncertainty. However, for estimated models with a well-defined posterior distribution for parameters, accounting for parameter uncertainty is straightforward. See Warne, Coenen, and Christoffel (2013) for discussion of predictive densities and predictive likelihood computations.

${ }^{5}$ In the text, the terms Gaussian and Normally-distributed are used interchangeably.

${ }^{6}$ The term "embarrassingly parallel", or "naturally parallel", is used for problems that are easy to parallelize because the sub-tasks can run independently of the others, without the need for sub-tasks to communicate with each other or synchronize.
} 


\section{Estimating the Structural Economic Shocks with Non-Linear Models}

The historical analysis boils down to finding the initial conditions $x_{0}$ and the path of structural economic shocks $\left\{\varepsilon_{t}\right\}_{t=1}^{T}$ such that the model dynamics of the observed variables $y_{t}$ matches the data. This is an "inversion" problem and, in general, the inversion problem requires a specification of the distribution of the structural shocks, $\left\{\varepsilon_{t}\right\}$.

In general settings, a distribution of shocks must be specified to estimate the path of the shocks, even if the higher-order moments are not needed to solve or simulate the model. In the linear setup when Gaussian shocks are assumed, the shocks are usually estimated with the use of the Kalman filter.

\section{“One-to-one": an important special case}

In the special case, where the initial conditions $x_{0}$ are given and the number of observed variables equals the number of structural shocks to estimate, it is possible to estimate the path of structural shocks by solving the model "in reverse" as a system of nonlinear difference equations. Essentially, one solves an implicit problem $\varepsilon_{t}=f^{-1}\left(x_{t-1}, y_{t} ; \theta\right)$ for each period, using the estimated shocks to update the aggregate state of the economy. In this important special case, the distribution of shocks has no role for the solution and is not needed to estimate the path of the shocks. Variations on the "one-to-one" case are easy to accommodate, with estimation of the initial conditions. ${ }^{7}$ See the appendix for details.

The "one-to-one" assumption is not unusual. Most of the existing DSGE models have the same number of stochastic shocks as observed variables, including the canonical models by Smets and Wouters (2007), with 7 observable variables and 7 shocks, or Christiano, Motto, and Rostagno (2014) who have 12 observed variables and 12 stochastic shocks.

After the path of the shocks is estimated, their statistical properties can be analyzed to assess model-specification. However, if the model is used for risk assessment the estimated shock distributions are used to run stochastic simulations to estimate the impact of the distribution of the historical data on the uncertainty around the forecast path.

\section{Distribution of Shocks and Resampling}

Considered here is the case of the "one-to-one" estimation of the shocks using the model and observed data, where the paths of all shocks that replicate the data are obtained without strong assumptions about the distribution of the shocks. The starting point for distribution

\footnotetext{
${ }^{7}$ It is also straightforward to calculate the likelihood function for a non-linear model using this "one-to-one" inversion approach, if the distribution of shocks is specified. With long data sample the effects of uncertainty about the initial conditions will fade away quickly for stationary variables.
} 
estimation is then an $(R \times T)$ matrix of shocks $E=\left[\varepsilon_{1}, \varepsilon_{2}, \ldots, \varepsilon_{T}\right]$. The column vectors $\varepsilon_{t}$ are all the economic shocks for the current period (multiple shocks for multiple regions).

To sample from the unknown distribution of $\varepsilon_{t}$, the simple solution would be to bootstrapsample with replacement - from the estimated shocks by resampling from the time index $\mathrm{t}=1, \ldots, T$ to preserve the cross-sectional dependence among the $R$ elements in $\varepsilon_{t}$. The issue with this solution is impoverishment - there only $T$ periods to resample. To avoid the problem, each of the re-sampled shocks, $\varepsilon_{t, s}$, can be augmented with "jitter" - a random noise to effectively enrich the sample. It turns out there is a close connection between this intuitive bootstrap approach and sampling from the kernel density estimate.

\section{Kernel Density Estimation and Sampling}

A simple and agnostic approach to estimate the joint distribution of shocks nonparametrically is using kernel density estimation (KDE) techniques, see $\mathrm{Li}$ and Racine (2007). While not often described, it is straightforward to sample from the estimated kernel density (from a "smoothed histogram").

Sampling from the KDE is a two-step process:

i. Draw a point $\varepsilon_{t}$ from all the available shock estimates in $E$ (with replacement).

ii. Given the point $\varepsilon_{t}$, draw from the kernel $K\left(\varepsilon_{t}, \Sigma\right)$ with the estimated bandwidth matrix, $\Sigma$. For instance, if the Normal kernel is used, this amounts to drawing from Normal distribution with the mean $\varepsilon_{i}$ and the covariance matrix $\Sigma .8$

Intuitively, sampling from the kernel density estimate can be understood as uniformly resampling the existing data points, $\varepsilon_{i}$ with an added "jitter", a noise to "rejuvenate" the draws. The narrower the jitter bandwidth, the less smoothing. For the stochastic simulations, history does repeat itself, or it at least "rhymes".

\section{Generalized Bootstrapping}

With non-trivial dependence among the shocks in time as well as cross-correlation, a more general sampling setup can be used. The idea of bootstrapping is resampling from the empirical distribution function, see Efron (1979), or Künsch (1989) for the case of bootstrap for data with complex dependence patterns

Given the large number of shocks in the models, the full multivariate kernel density estimation can be infeasible. An alternative is to sample from the empirical cumulative

\footnotetext{
${ }^{8}$ In this case, it is convenient to separate the "size", $h$, from the "orientation", A, i.e. $\Sigma=h^{2} A$. It is the estimation of the bandwidth matrices that is the challenging step in the KDE estimation, see Li and Racine (2007). There are multiple "rules of thumb" approaches, as well as an extensive use of cross-validation to determine the appropriate bandwidth. Given our interest in sampling from the KDE, the sensitivity to finding the "right" bandwidth is smaller.
} 
distribution function (ECDF) using a $k$-block bootstrap, where $k$ denotes the number of periods. For example, for a forecast horizon of five years, it is feasible to draw from five-year histories of the shocks. To avoid the degenerate sample, a "rejuvenation" step is needed as in the case of KDE sampling, while keeping the variance stable.

\section{Weighted Sampling}

Sampling based on a set of estimated shocks also allows for non-trivial weighting of the information in the $(R \times T)$ matrix of shocks, $E$. For instance, the sample can be restricted only to selected years - 1962-today or 1992-today, corresponding to zero weights for selected years during the resampling step. Alternatively, selected years may be given a zero weight to investigate their effect on the predictive distribution - for instance, one can exclude the recessions years.

Another option to weighted sampling is to reflect different probability of shocks to be drawn based on the current state of the business cycle, possibly reflecting the information not included in the model. For instance, the probability weights could be higher for shocks historically occurring at or near the level of the current value of the output gap, or a financial conditions index.

More parametric approaches to estimate the shock distributions are feasible and may be suitable for different sample sizes, e.g. multi-variate skewed Normal distribution, tdistribution, Gaussian mixtures, etc. The KDE/bootstrap sampling, however, is convenient fast, and very general since no closed-form description of the shock distribution is needed.

\section{Providing Globally-Consistent Risk Assessment}

\section{A. Assumptions}

The examples in the paper use the G20MOD module from the IMF's Flexible System of Global Models (FSGM). G20MOD a large-scale semi-structural model covering the whole world economy divided into 25 countries/regions. This includes blocks for each G-20 country with the remaining countries aggregated into the larger regions. ${ }^{9}$ See Andrle et al. 2015 for the details on other modules.

The model represents the whole world and the international linkages are through trade and financial flows. Each country is described by a behavioral structure driving the dynamics of the components of real GDP, core and headline inflation, wages, unemployment, the current

\footnotetext{
${ }^{9}$ All computations in this paper are based on a code base with sub-modules written in Troll, Fame, Matlab, and Python. All the computations are heavily parallelized into multiple "jobs". One draw, consisting of a sequential five-year horizon simulation, takes at minimum 15 minutes per worker.
} 
account, fiscal revenues and expenditures, debt, the deficit and many other macroeconomic variables.

The illustration will showcase risk assessment around a baseline forecast. For this illustration, only the data for real GDP, potential output, and the cyclical dynamics in oil prices are used to estimate the global oil prices shock, individual country demand shocks, or the shocks to potential output growth. The sample ranges from 1960 to 2018.

In principle, it is desirable (and technically easy) to include other observable macroeconomic variables, such as inflation, interest rates, or the structure of GDP for the estimation of the shocks, as long as the sampling acknowledges the possible dependence among the shocks. In the current application, the probability distribution of headline inflation in our example application disregards cost-push shocks, exchange rate shocks, or inflation target credibility shocks, relevant for many countries. For this exercise to the goal is to illustrate the power of the co-movement among variables that demand shocks generate in G20MOD.

A U.S. demand shock is illustrated in Appendix Fig. 6 for convenience. A positive demand shock boosts both consumption and investment, inflation rises, and unemployment rate declines. The co-movement properties of the demand shock are crucial for empirical performance of the model and correspond well to empirical evidence suggesting that one dominant shock (factor) can explain large portion of the cyclical dynamics of key macro variables, see Andrle, Bruha, and Solmaz (2017). Figure 77 in the Appendix illustrates that one estimated factor accounts for more than 80 percent of dynamics of output, consumption, investment, imports, and unemployment. Figure 88 illustrates that the estimated path of inflation and interest rates in the U.S., conditioned on the estimated demand shocks and the variables' trend dynamics, aligns well with the data.

The paths of the shocks were estimated using the "one-to-one" approach, by endogenizing selected shocks and making exogenous the variables for which observations are available. The estimated shocks serve as an input to the estimation of their joint empirical cumulative distribution functions (ECDF), which will be used to generate the counterfactual scenarios. The $k$-periods bootstrap is used to sample shocks from the ECDF which are then used to simulate the model. Resampling from the kernel density estimates, or one-period bootstrap yield similar results. 


\section{B. Predictive Distributions around the Baseline Forecast}

The interpretation of the results must reflect the specific assumptions above, as well as the structure of the model. The estimated distributions of the GDP growth, and other variables, are not Gaussian - they are asymmetric, skewed towards the downside. There are two key reasons for this:

a) the estimated economic shocks are asymmetric, and

b) the model is not linear (with a key non-linearity being the effective lower bound (ELB) on nominal interest rates.

\section{Shock Distributions}

The marginal distributions of the estimated shocks are not Gaussian and are asymmetric with fat tails (leptokurtic). The kurtosis of the demand shocks and oil price shocks ranges from 36 , while the kurtosis of a univariate Normal distribution is 3. The marginal distributions are also negatively skewed, with the median skewness of -.65 and the minimum of -1.7. This is due to several sharp contractions in the data from 1960-2018. Fig. 1 displays the quantilequantile "qq" plots comparing the quantiles of two estimated demand shocks with the Normal distribution, with clear signs of a heavy left tail in the marginal distribution. The estimated shocks also display time-varying co-movement, with one of the largest synchronizations of demand shocks occurring in 2008.

Reflecting the kurtosis and skewness of the shock distribution is vital for specifying realistic shock distributions and model-based risk assessment. Even propagating non-Gaussian shocks through a linear economic model would help to better assess forecast risk.

\section{Non-linearity}

There are two sources of non-linearity in the model- the non-linear functional form of the model's equations, and the implemented "effective lower bound" (ELB) on nominal interest rates. While many state-of-the-art DSGE models are close to log-linear the effective lower bound is an important time-varying non-linearity.

For each country, the distance to its presumed effective lower bound will define the available monetary-policy space. With no or limited monetary policy space, the impact of negative economic shocks is exacerbated, with deeper and longer output declines.

\section{Output Distributions and "Fan Charts"}

Figure 2 illustrates the estimated distribution of output growth at a one-year and five-year horizon for a list of G-20 economies and the World (WRL), with the mode accentuated by the vertical line for the one-year horizon distribution. The width of the distribution, and thus 
the uncertainty, widens as the horizon increases. The shift in the mode depends on the country's current and projected position in the business cycle.

Figure 3 illustrates the forecast "fan chart" for key macroeconomic variables (output growth, headline inflation, short term interest rate, and output level) for Japan. Based on these distributions, the probability of negative output growth, or the probability of deflation can be estimated.

To illustrate the flexibility of the weighted sampling, Figure 4 shows the marginal distribution of output growth in the U.S. based on the full sample, compared to a sample where the shocks around U.S. recessions are excluded. ${ }^{10}$ Not surprisingly, when the recessions are removed, the left tail of the distribution changes significantly, while the distribution of the positive-growth surprises stay largely unaffected. Similarly, the output growth distribution is narrower for the sample truncated to the post 1992 period as opposed to the full 1962-2018 sample.

The last application example in Table 1 illustrates the implied probability of world output growth one-year-ahead turning out to be less than 2.5 percent at three different time periods, corresponding to three World Economic Outlook (WEO) vintages. This exercise is similar to the one in the IMF's October 2019 WEO (IMF 2019) which were based on using G20MOD under the methodology described in this paper. For an alternative and fully non-parametric approach to global growth risk assessment, see Caselli et al. (2020), who construct the predictive growth density using conditional kernel density estimation at quarterly frequency.

\footnotetext{
10 The model and all the coefficients are kept unchanged.
} 
Figure 1: Sample Shocks vs. the Normal Distribution
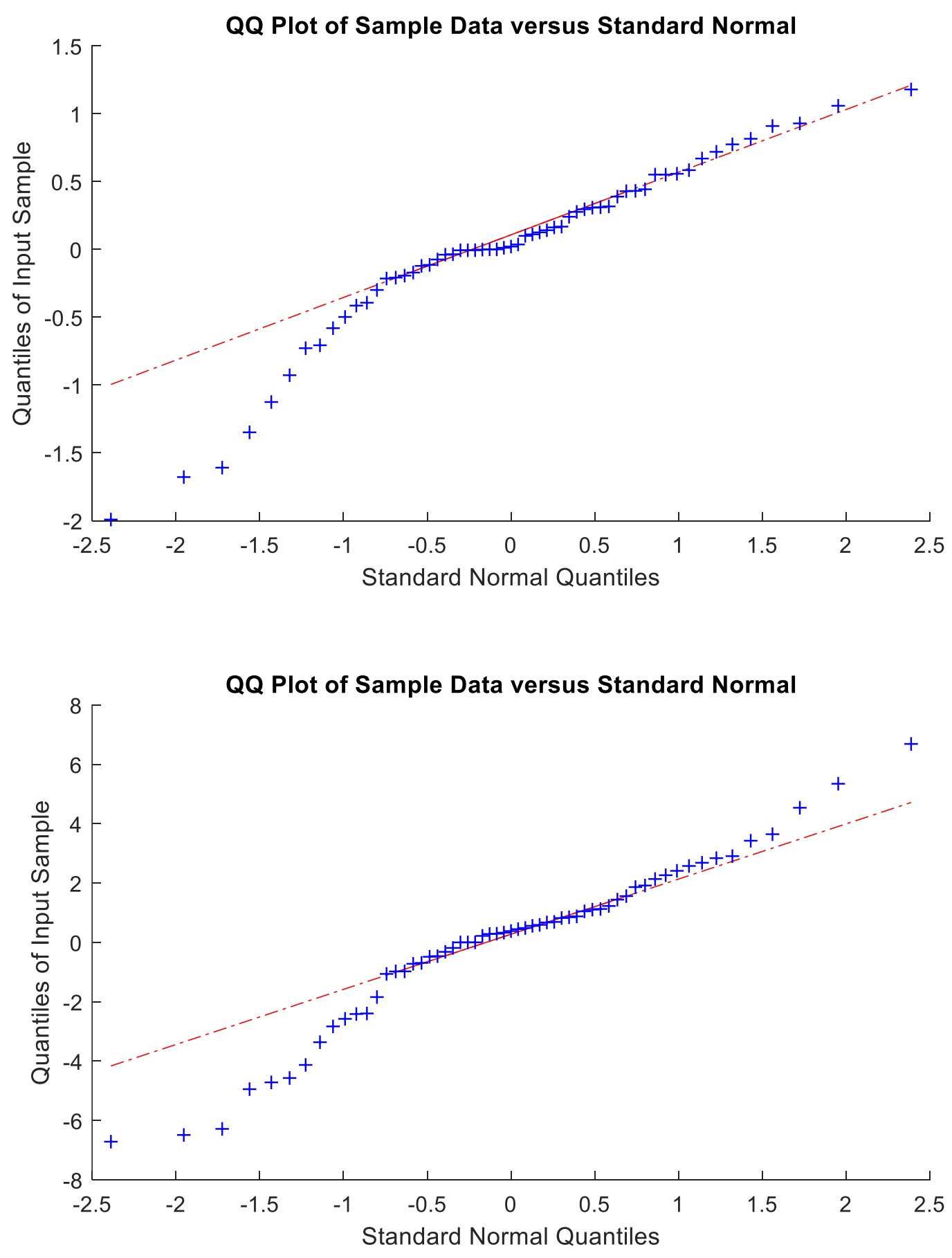
Figure 2: Estimated GDP Growth Distribution (1Y and 5Y horizon)
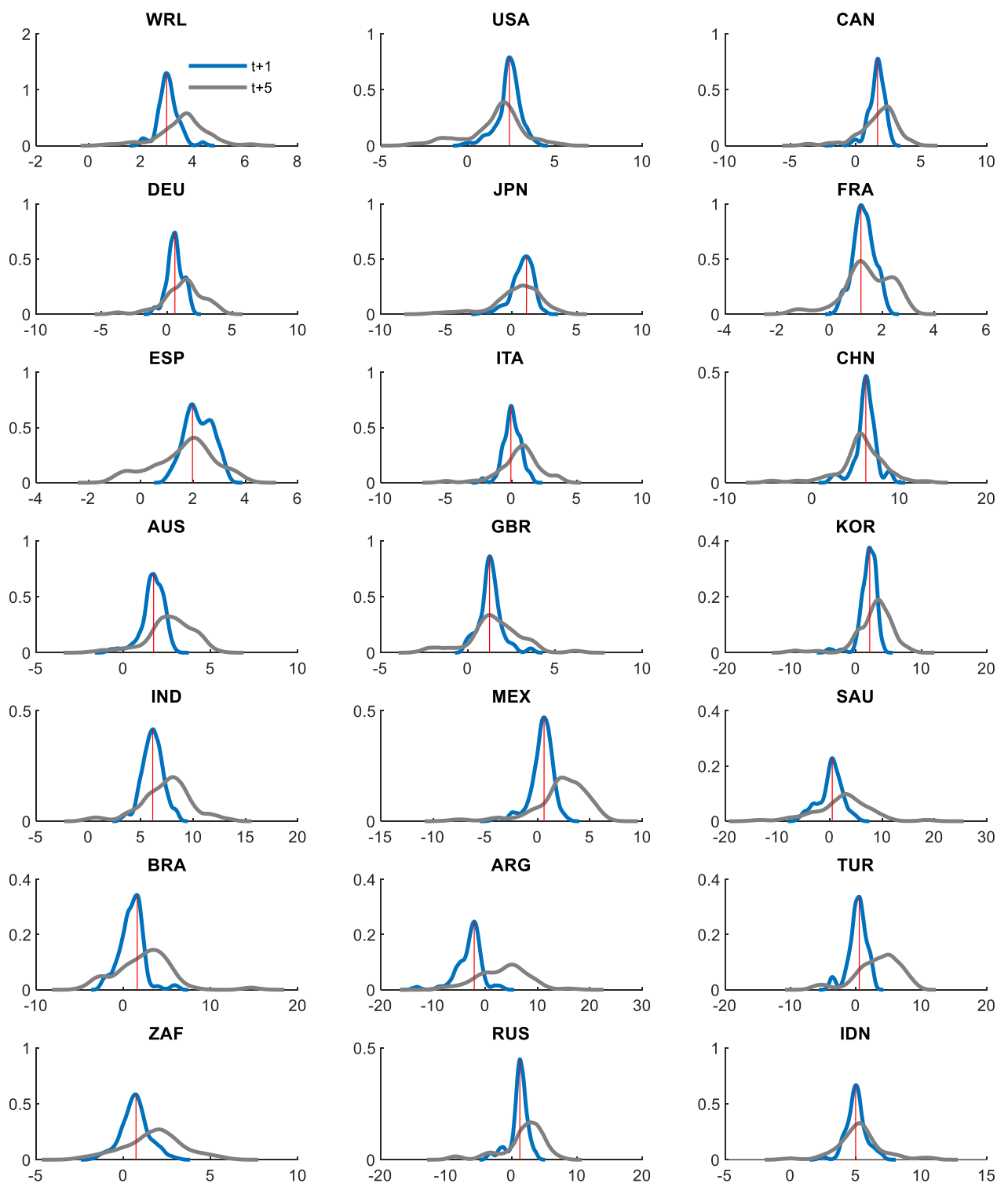
Figure 3: Fan Charts around the Baseline Forecast (5-95 percentiles): Japan
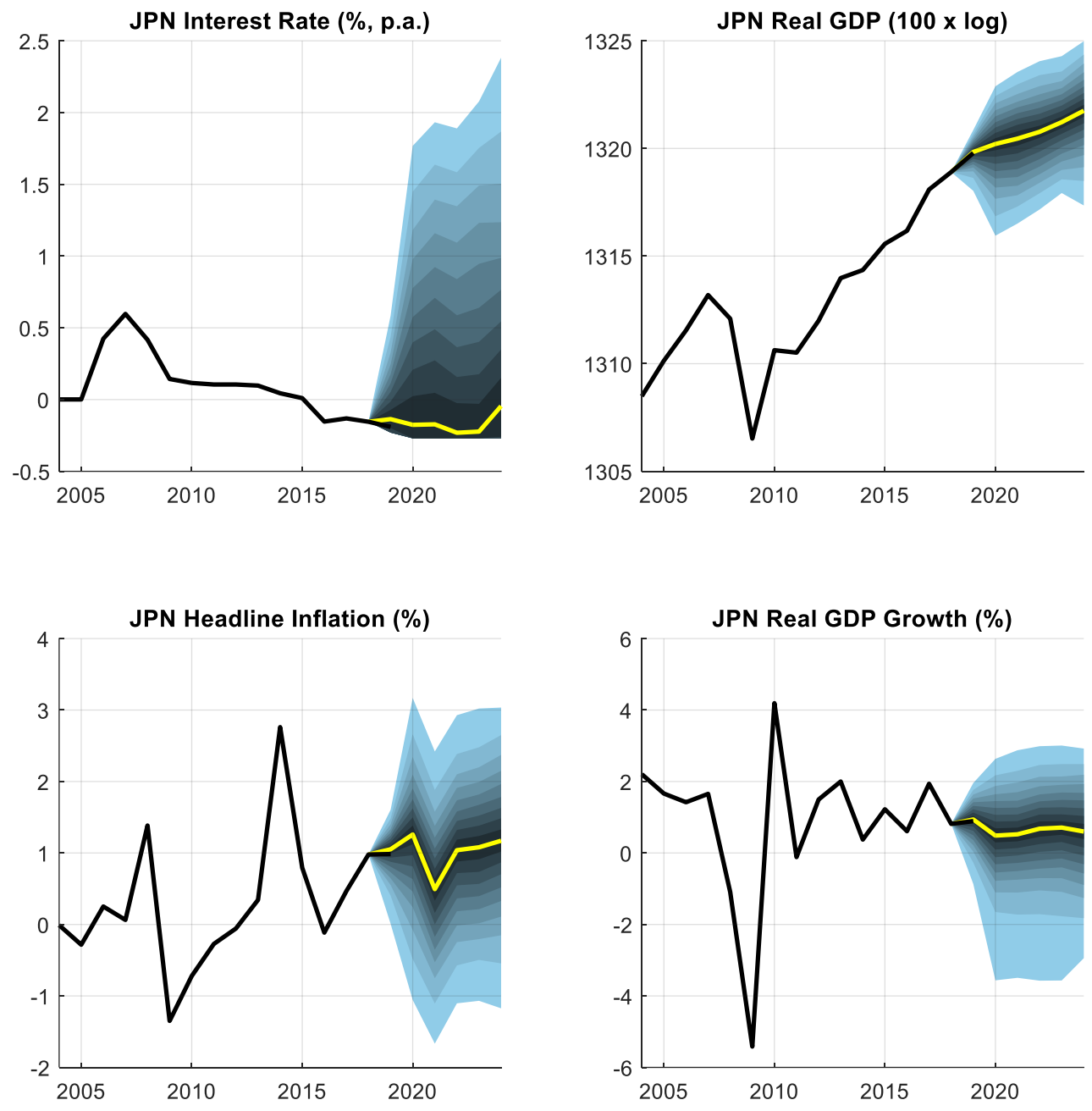
Figure 4: Marginal Distribution of the GDP Growth in the U.S.

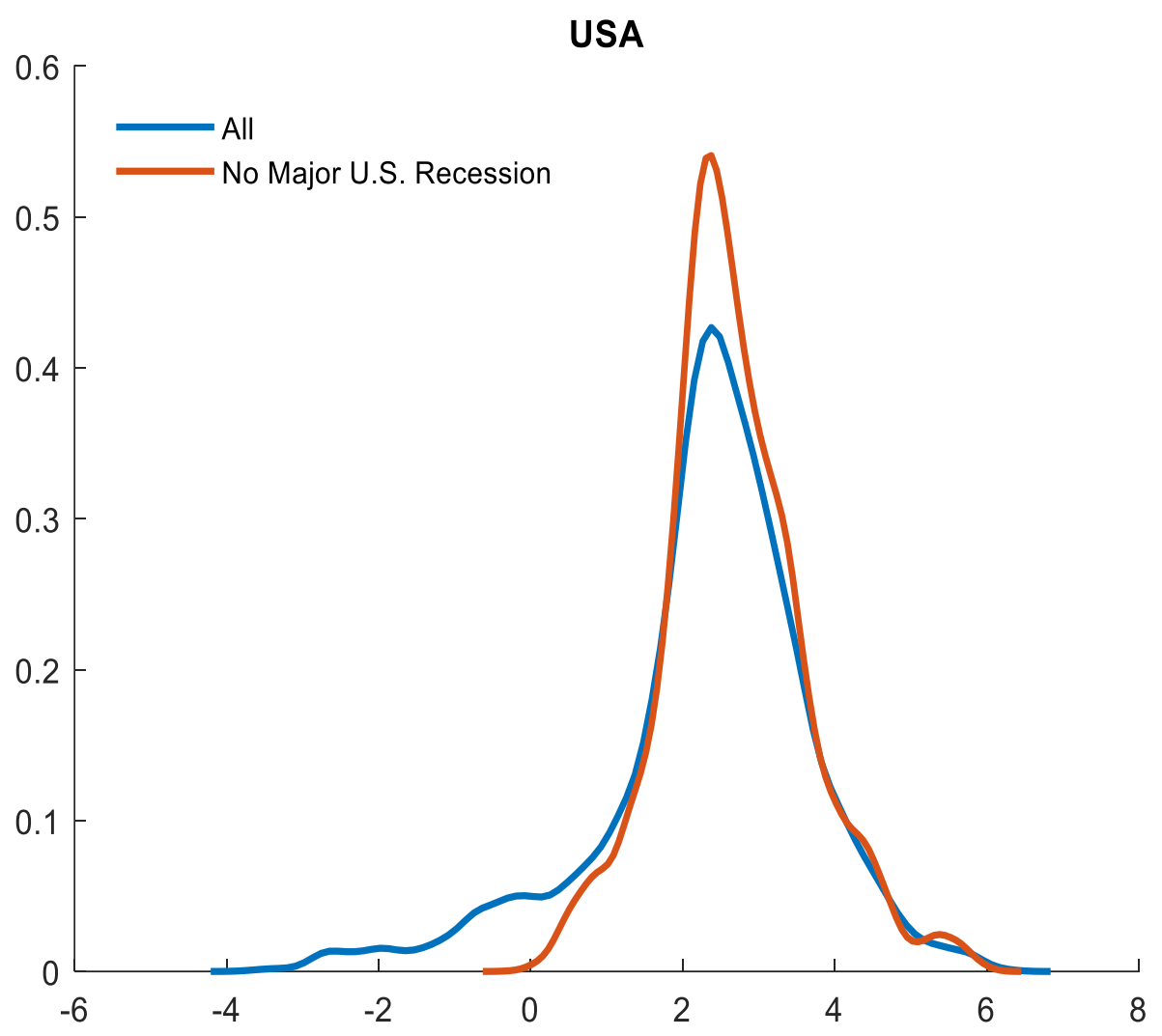

Table 1: Probability of World GDP Growth of less than 2.5 Percent

\begin{tabular}{|l|c|c|}
\hline Assessment* & Full sample: 1962+ & Smaller sample: 1992+ \\
\hline October 2019 & 8.9 & 7.5 \\
\hline April 2019 & 7.8 & 5.9 \\
\hline October 2018 & 7.6 & 5.7 \\
\hline
\end{tabular}

Note: * pseudo real-time using the assumptions in this paper and vintage IMF WEO data 


\section{Effects of Alternative Economic Policies on Predictive Distributions}

Another important use of the technique discussed in this paper is an assessment of alternative economic policy options. The example below shows that automatic fiscal stabilization policy can be very effective if the economy is at or close to its effective lower bound (ELB) on nominal interest rates.

Figure 5 illustrates a one-year ahead assessment of risk for two alternative policy options in a representative advanced economy. Its baseline growth is 2.3 percent and the policy rate is close to the effective lower bound, with only 25 basis points of policy space. For simplicity, it is assumed no other form of monetary policy easing is being implemented. Based on the empirical distribution of past shocks and their variance, this example uses symmetric, Normally-distributed innovations. Any resulting asymmetry is due to the non-linear nature of the model and the occasionally-binding effective lower bound (ELB) on nominal interest rates. ${ }^{11}$

In Figure 5, the base case represents a standard operation of the monetary and fiscal policy rules, when there are no constraints on the monetary policy reaction. The first alternative scenario introduces a restriction that the current policy cannot decline more than 25 basis points, thus severely limiting the available monetary policy space. Given the lack of monetary policy space, the Normally-distributed risks for the economy translate into a negatively-skewed growth distribution. Fiscal policy is not active beyond the default, and relatively weak, automatic stabilizers included in the standard fiscal reaction function.

The second alternative case assumes that the government implements an active fiscal policy rule with explicit macro triggers. The calibration is such that if the unemployment rate increases by a half percentage point beyond a pre-specified threshold (an estimate of the long-run trend or natural rate of unemployment), a transfer to households equivalent to 0.7 percent of GDP is executed. The rule is asymmetric and there is no decline in transfers in the boom period when unemployment falls below trend. The design and calibration of the rule is inspired by — but not identical to — the rule proposed by Sahm (2019).

These simulations illustrate that with an active, asymmetric fiscal policy reaction function, the probability of a severe downturn may be significantly diminished. Given the fiscal boost during severe downturns, the distribution of the debt-to-GDP ratio is more adverse than when monetary policy is unconstrained, and no additional fiscal boost is implemented beyond a standard symmetric countercyclical fiscal reaction. However, the asymmetric aggressive fiscal rule clearly dominates in terms of output variability relative to the case when the effective lower bound is frequently binding and fiscal policy follows a conventional symmetric countercyclical reaction function.

\footnotetext{
${ }^{11}$ For results using the shocks drawn from the empirical distribution function see Fig. 9 in the Appendix.
} 
Figure 5: Effects of Economic Policy on Risk Assessment
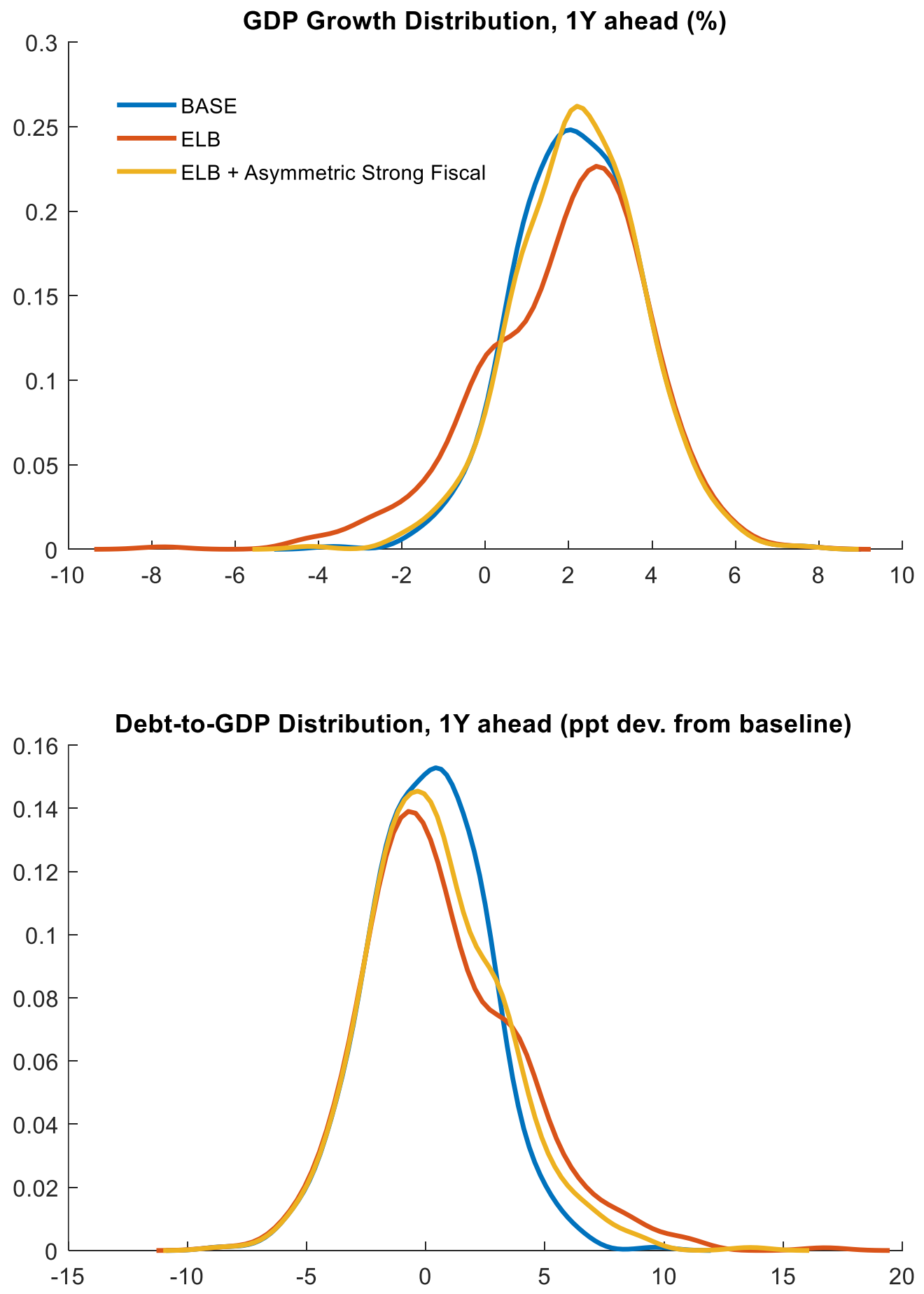


\section{CONCLUSION}

This paper details a practical approach to globally-consistent risk and policy assessment using non-linear global economic models. The risk assessment relies on an estimated predictive density around the baseline forecast.

Given an estimated predictive distribution for all the model's variables in all countries and regions it is feasible to quantify the probability of recessions, deflation, or the probability of hitting the effective lower bound on nominal interest rates, for instance. Further, different economic policies can be assessed by their impact on the distribution of output, inflation, and other model variables.

The contribution of this paper lies mainly in providing guidance on how to estimate shocks with large-scale non-linear models and allow for non-trivial statistical distributions for those economic shocks. Due to both the model's non-linearity (e.g. interest rate distance to their effective lower bound) and the estimated non-Gaussian distribution of shocks, the resulting risk assessment is asymmetric, skewed to more adverse outcomes in the extreme tails of the distribution.

The empirical illustrations use the IMF's G20MOD to showcase the predictive distributions around a baseline forecast and the statistical properties of the estimated economic shocks. One example also shows how the distribution of output changes with alternative fiscal policy rules, when the economy is close to its effective lower bound on nominal interest rates.

The approach developed in this paper is simple and agnostic about the structural shocks' distribution. The distribution is shaped by the empirical estimates of the shocks using a given model. The draws from the shocks' distributions are then used to generate counterfactual scenarios for risk assessment. 


\section{REFERENCES}

Andrle, M., Patrick Blagrave, Pedro Espaillat, Keiko Honjo, Benjamin Hunt, Mikko Kortelainen, Rene Lalonde, Douglas Laxton, Eleonora Mavroeidi, Dirk Muir, Susana Mursula, and Stephen Snudden, 2015, "Flexible System of Global Models - FSGM," IMF WP/15/64.

Andrle, M. Jan Brůha, and Serhat Solmaz, 2017, "On the Sources of Business Cycles: Implications for DSGE models," European Central Bank Working Paper No 2058.

Carabenciov, I., Charles Freedman, Roberto Garcia-Saltos, Douglas Laxton, Ondra Kamenik, and Petar Manchev 2013, "GPM6-The Global Projection Model with 6 Regions," IMF WP/13/87.

Christiano, L.J., R. Motto, and Massimo Rostagno, 2014, “Risk Shocks,” American Economic Review, 104(1), pp. 27-56.

Caselli, F., F. Grigoli, R. Lafarguette, and Ch. Wang, 2020: "Predictive Density Aggregation: A Model for Global GDP Growth", IMF Working Paper WP/20/XX [forthcoming].

Efron, B., 1979, "Bootstrap Methods: Another Look at the Jackknife," The Annals of Statistics, 7, pp. 1-26.

IMF, 2019, "World Economic Outlook - Chapter 1: Global Manufacturing Downturn, Rising Trade Barriers, “IMF WEO October, 2019, pp. 22 and Fig. 1.23.

Juillard, M, 1996, "Dynare: A program for the resolution and simulation of dynamic models with forward variables through the use of a relaxation algorithm", CEPREMAP Working Paper (Couverture Orange), 9602.

Kumhof, M., Douglas Laxton, Dirk Muir, and Susanna Mursula, 2010, "The Global Integrated Monetary and Fiscal Model (GIMF) — Theoretical Structure", IMF $\mathrm{WP} / 10 / 34$.

Künsch, H.R., 1989, "The Jacknife and the Bootstrap for General Stationary Observations," The Annals of Statistics, 34, pp. 1217-1241.

Li, Q. and Jeffrey S. Racine, 2007, "Nonparametric Econometrics: Theory and Practice," Princeton University Press.

Morley, J. and Jeremy Piger, 2012, "The Asymmetric Business Cycle," The Review of Economics and Statistics, Vol. 94 (1), pp. 208-21. 
Sahm, C., 2019, "Direct Stimulus Payments to Individuals," in "Recession Ready: Fiscal Policies to Stabilize the American Economy," (eds.), Heather Boushey, Ryan Nunn, and Jay Shambaugh.

Smets, F. and Rafael Wouters, 2007, "Shocks and Frictions in US Business Cycles: A Bayesian DSGE Approach,” American Economic Review, Vol. 97, No. 3, June 2007.

Warne, A., Gunter Coenen, and Kai Christoffel, 2013, "Predictive Likelihood Comparisons with DSGE and DSGE-VAR Models,” ECB WP No. 1536, April 2013. 


\section{APPENDIX}

\section{Figure 6: Impulse-Response Function for US Demand Shock}
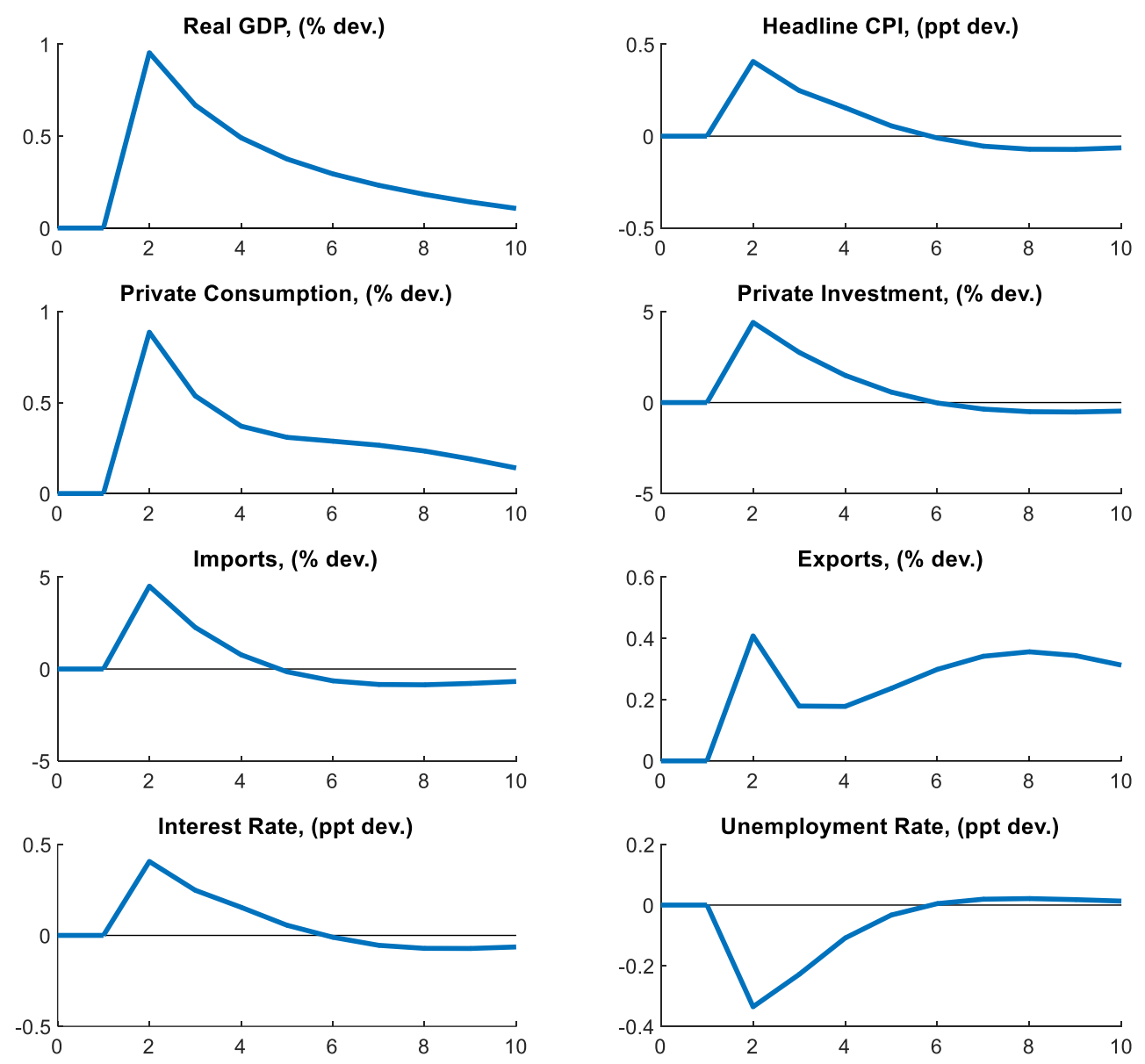
Figure 7: Cyclical Component of US Macro Data
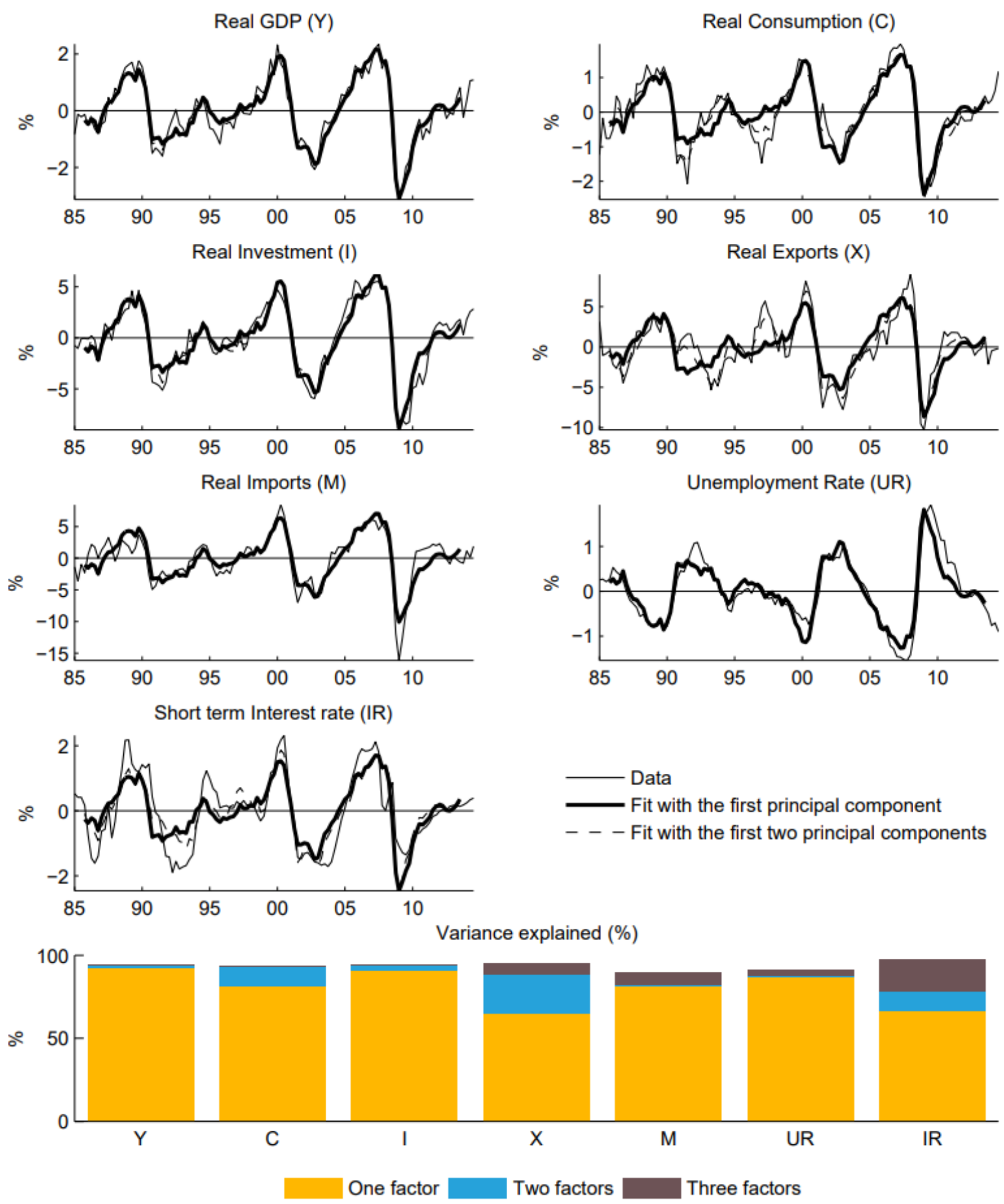

Source: Andrle, Bruha, and Solmaz (2017)

Note: Cyclical components of variables and their fit using one and two principal components, with constant loading factors. 
Figure 8: Model-implied vs. Actual Outcome
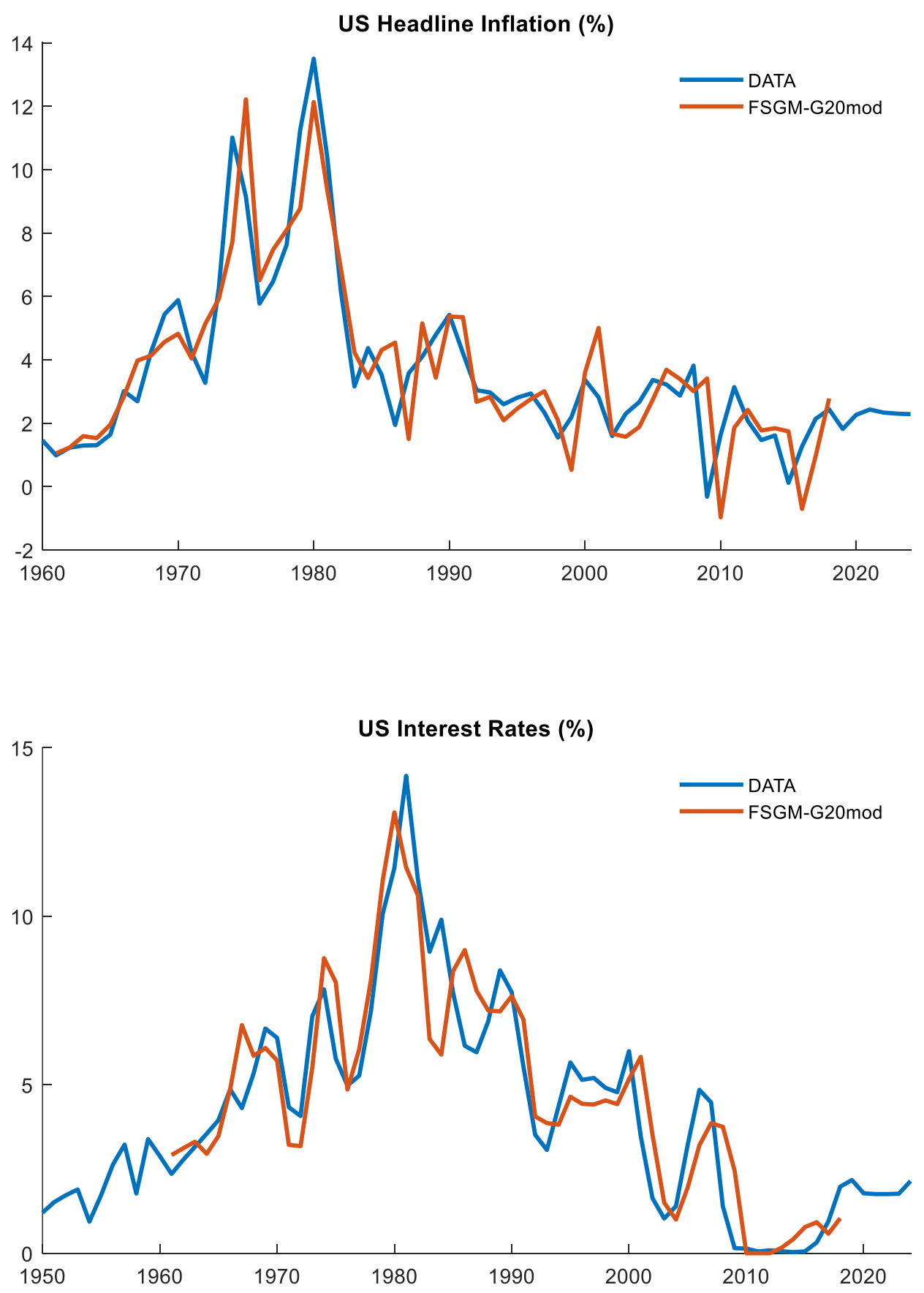

Note: FSGM simulations conditioned on demand shocks and oil price shock only, with inflation target and trend in real rate being exogenous. The data include the IMF WEO forecast up until 2025 as of October 2019. 
Figure 9: Distribution of GDP Growth and Debt-to-GDP using the Empirical Shock Distribution
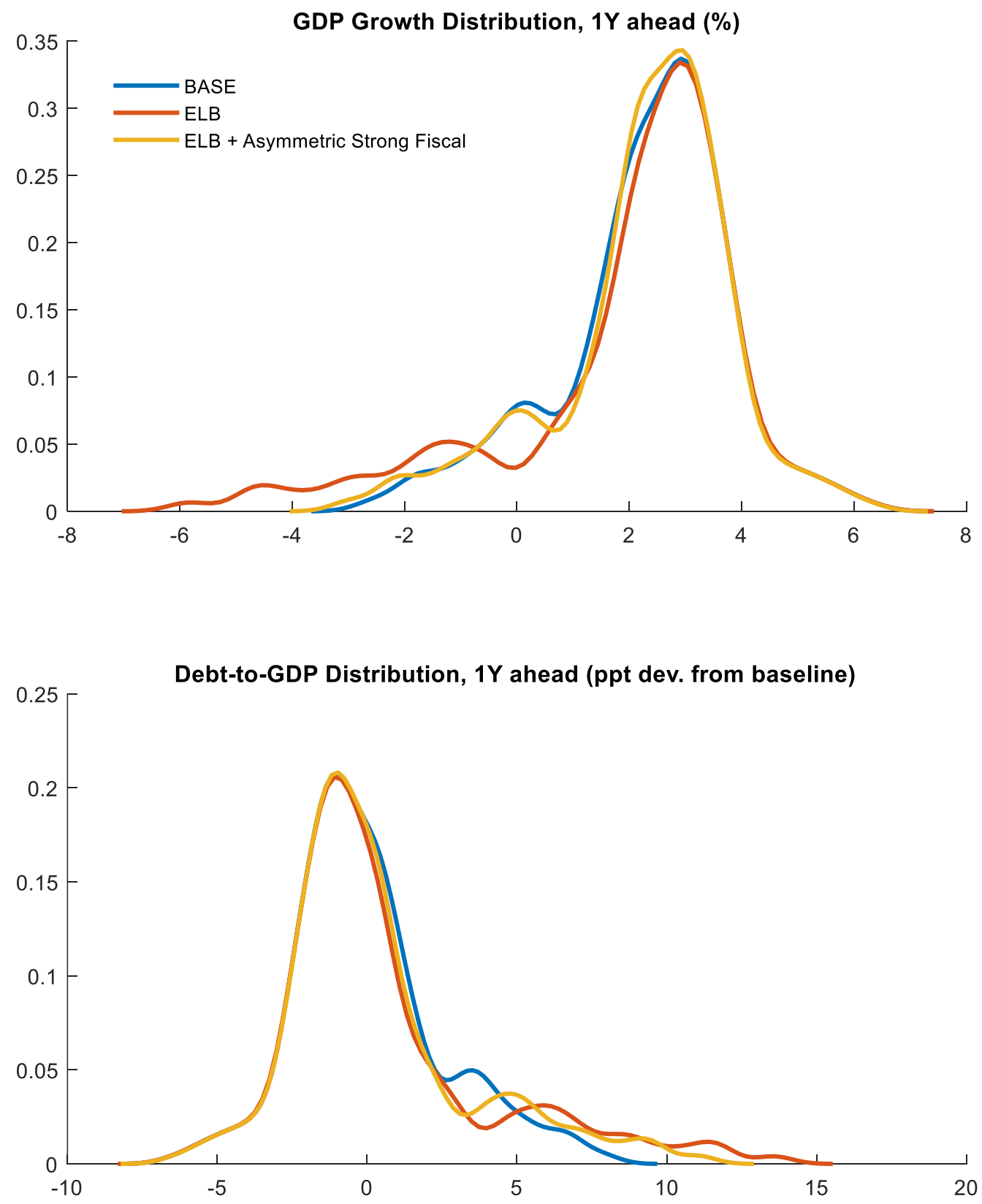


\section{A. Stack-Time Solution Algorithm: Intuition}

It is useful to understand the stack-time solution algorithm used to solve the non-linear model and the estimation of the shocks for the stochastic simulations.

The model, in its certainty-equivalent form, is written as follows:

$$
\left.M\left(x_{t+1 \mid t}, x_{t}, x_{t-1}, \varepsilon_{t} ; \theta\right)\right]=0
$$

In principle, if $x_{t+1 \mid t}$ was known, solving for $x_{t}$ is a static, nonlinear problem. However, for both transitory shocks or permanent shocks, it is known what the expectations are in the long run (say in 200 periods). For a transitory shock, the long-run solution is the steady state of the model, for permanent shocks it is the new steady state of the model.

\section{Stack-Time Solution}

Given the recursive nature of the system, the model can be re-written as a static system of equations, one for each period. The trick is to keep the equations "stacked in time":

$$
\begin{gathered}
\left.M\left(x_{2 \mid 1}, x_{1}, x_{0}, \varepsilon_{1} ; \theta\right)\right]=0 . \\
\left.M\left(x_{3 \mid 1}, x_{2 \mid 1}, x_{1}, \varepsilon_{2 \mid 1} ; \theta\right)\right]=0 . \\
\left.M\left(x_{4 \mid 0}, x_{3 \mid 1}, x_{2 \mid 1}, \varepsilon_{3 \mid 1} ; \theta\right)\right]=0 .
\end{gathered}
$$

The current example considers only the periods $t=1,2,3$, with the initial condition $x_{0}$ in $t=0$ assumed known and given. It is also assumed that the expected path of shocks is given, conditional on the current information set in $t=1$. (Variables in red are given).

Now, should also the "terminal condition" would be known, which is $x_{4 \mid 0}$ in our example, then there are three sets of nonlinear equations to solve for the three sets of variables: $x_{1}$, $x_{2 \mid 1}, x_{3 \mid 1}$, given the parameters, the initial conditions, and the expectations of shocks.

If a long-enough horizon for the terminal condition is assumed, then solution to such an extended system is valid. It is, however, important to check that the results are not sensitive the period at which the terminal condition is imposed. The example with terminal condition in the fourth period is too short and only for illustration. As already mentioned, for transitory shocks the terminal conditions will just be the current steady state of the model, whereas a new steady state will be used for a terminal condition with permanent shocks. For technical details about the stack time solution, see Juillard (1996). 


\section{Shock Estimation}

When the "one-to-one" estimation of the shocks is used in this paper, the system of equations above is solved with additional twist. Instead of solving for $x_{1}$, given the shock $\varepsilon_{1}$, the variables are considered as given, set to their data values, and the shocks required for the solution, $\varepsilon_{1}$, are endogenously solved for. This solution is repeated every time period, to solve for the unexpected shocks consistent with the data. This approach is also used to produce judgmental forecast, with variables conditioned on expert judgment, for instance. 